Revue des patrimoines

\title{
Comprendre la technique des peintures romanes par le relevé stratigraphique
}

\section{Carolina Sarrade}

\section{(2) OpenEdition}

1 Journals

Édition électronique

URL : http://journals.openedition.org/insitu/10641

DOI : 10.4000/insitu. 10641

ISSN : 1630-7305

Éditeur

Ministère de la Culture

\section{Référence électronique}

Carolina Sarrade, "Comprendre la technique des peintures romanes par le relevé stratigraphique », In Situ [En ligne], 22 | 2013, mis en ligne le 15 novembre 2013, consulté le 14 novembre 2019. URL http://journals.openedition.org/insitu/10641 ; DOI : 10.4000/insitu.10641

Ce document a été généré automatiquement le 14 novembre 2019.

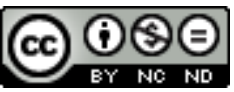

In Situ Revues des patrimoines est mis à disposition selon les termes de la licence Creative Commons Attribution - Pas d'Utilisation Commerciale - Pas de Modification 4.0 International. 


\title{
Comprendre la technique des peintures romanes par le relevé stratigraphique
}

\author{
Carolina Sarrade
}

Communication présentée lors des journées d'études consacrées à l'Actualité de la recherche en peinture murale, organisées par le musée des Monuments français les 25 et 26 octobre 2011, à la

Cité de l'architecture et du patrimoine.

\section{Méthode d'analyse}

1 Depuis environ un siècle, les méthodes de relevé des peintures, et notamment celles qui s'inscrivent dans le contexte monumental, ont évolué vers une recherche de restitutions toujours plus fidèles répondant aux exigences d'une documentation de plus en plus précise nécessaire à la conservation du patrimoine. Dans un premier temps, les techniques employées sont demeurées relativement sommaires, rejoignant celles qui étaient utilisées pour d'autres époques : décalque rapide des images pariétales, frottis à la mine de plomb des gravures, aquarelles ou dessin à main levée pour les peintures sous abris.

Depuis 1999, la technique du relevé stratigraphique des peintures murales, conçue sur le chantier de la crypte de Saint-Germain d'Auxerre, met à disposition du chercheur une nouvelle méthode d'analyse ${ }^{1}$. Suivant le principe utilisé pour les élévations en archéologie du bâti ${ }^{2}$, le relevé des surfaces peintes devient un témoin consultable à tout moment, moins fragile que la mémoire et permettant d'observer la stratigraphie des couches picturales qui peuvent correspondre soit aux différentes étapes de la mise en œuvre, soit aux interventions survenues dans le temps.

3 Ce travail soumet l'archéographe à une observation très rapprochée des parois, ce qui lui permet de noter un grand nombre d'informations dans divers domaines : technique 
utilisée, superposition des figures, état de conservation du support ou des pigments, repentirs. L'enregistrement systématique sur le calque des traces les plus ténues permet d'identifier des vestiges de peinture qui ont aujourd'hui presque entièrement disparu.

Lorsque l'ensemble du décor pictural est visible et qu'il existe des relations entre les différents motifs, le relevé est une méthode comparable à la phase d'enregistrement des données lors d'une fouille archéologique: chaque couche picturale correspond alors à une unité stratigraphique. Son analyse permet de constituer le diagramme stratigraphique de l'application des couleurs sur l'ensemble du programme et de proposer au lecteur une interprétation graphique qui rend compte de la complexité des faits observés. Les données archéologiques concernant l'ensemble d'un cycle peint et son évolution sont susceptibles de fournir de précieuses indications sur la fonction du monument et ses modifications.

Pour les peintures les plus dégradées, le relevé permet de proposer une restitution de leur état d'origine, combler certaines lacunes iconographiques, compléter les formes, restituer les surfaces colorées et homogénéiser l'ensemble en intervenant non pas sur la surface murale mais à travers une restitution informatique.

6 L'intervention débute par l'observation minutieuse à l'aide de loupes et sous un éclairage froid qui évite toute altération des pigments. Les traces de peinture parfois imperceptibles à l'œil nu sont codifiées et reproduites fidèlement sur un film plastique fixé dans les enduits de consolidation modernes ${ }^{3}$. Les calques sont ensuite numérisés, rassemblés et réduits sur ordinateur pour une reprise postérieure grâce à un logiciel de dessin vectoriel. Le programme informatique utilisé permet de travailler dans la gamme des couleurs, préalablement identifiées sur le terrain, grâce à un code de nuancier et reproduites sur le tirage papier. La phase du travail infographique aboutit à une restitution extrêmement fidèle des panneaux peints (fig. $\mathbf{n}^{\circ} \mathbf{1}$ ).

\section{Figure 1}

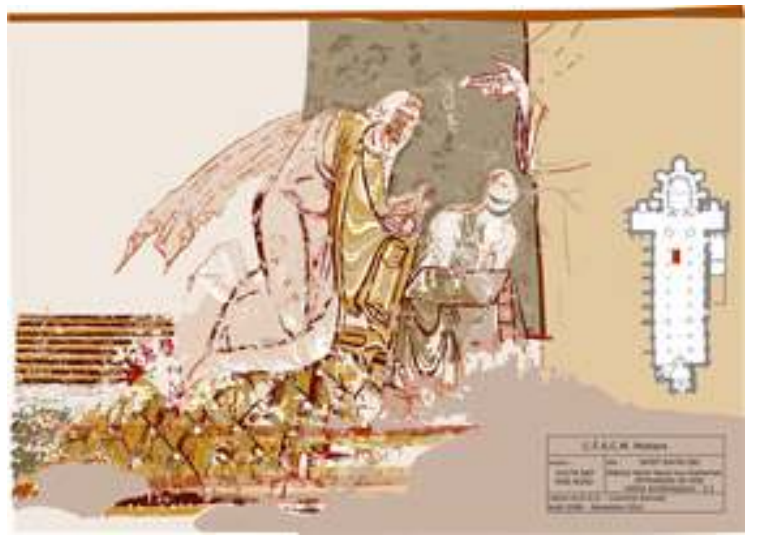

Saint-Savin-sur-Gartempe, peinture de la nef. Relevé stratigraphique du Sacrifice de Noé. Relevé Sarrade, Carolina. (C) CESCM, décembre 2011.

7 Le résultat de cette méthode présente l'avantage considérable de pouvoir décomposer le relevé en autant de calques qu'il y a d'étapes de mise en couleur, et de séparer les différents types de données (gravure, dessin préparatoire, aplat de couleurs, reliefs, incrustations, destruction du support). On peut facilement les sélectionner et, par exemple, ne visualiser que les esquisses initiales ou encore restituer virtuellement 
l'aspect d'un panneau avant les restaurations qui en perturbent parfois la lecture. Le relevé rend visible ce que l'usure et la distance des parois ne permettent plus de voir. Il facilite la lecture archéologique par la décomposition stratigraphique des peintures et donne la possibilité de les reproduire à la taille réelle.

Il permet au chercheur de prolonger son temps de réflexion, parfois contraint par la rapidité avec laquelle doivent s'effectuer les études de terrain. La précision des relevés permet de se livrer à une étude rigoureuse de l'objet archéologique sans être directement en contact avec celui-ci. Le temps que demanderaient toutes ces observations sur l'édifice ne permettrait pas de mener correctement le travail de compréhension globale des peintures.

Lors de la dernière campagne de restauration de la nef de l'abbaye de Saint-Savin-surGartempe, la problématique de l'équipe du CESCM résidait prioritairement dans l'identification de la technique utilisée, la proposition d'un résumé graphique des différents stades d'élaboration des peintures et la détermination d'une chronologie relative des décors dans l'ensemble de la nef.

Alors que les principaux ensembles iconographiques de Saint-Savin-sur-Gartempe sont bien connus et ont donné lieu à une importante bibliographie ${ }^{4}$, de nombreuses parties secondaires ont jusque-là été négligées. Certaines scènes soulevant des questions d'ordre iconographique ou archéologique ont donc été choisies pour être relevées à l'échelle 1.

11 Il est important de signaler que la réalisation systématique d'un relevé stratigraphique dans chaque travée n'aurait pas été utile. Néanmoins, pour les scènes n'ayant pas été relevées, une fiche analytique a été élaborée afin de constituer une grille d'observations permettant de compléter l'étude dans son ensemble et de mieux comprendre les changements relatifs aux décors picturaux.

12 À travers la réalisation des relevés, nous avons mis en évidence des stratigraphies et des pigments dont la compréhension réclamait des analyses chimiques complémentaires. Les observations ainsi faites ont permis de diminuer la quantité de prélèvements à effectuer, ceux-ci devant être aussi réduits que possible afin d'éviter de dégrader les peintures et les enduits ${ }^{5}$. Les prélèvements d'enduits, de peintures ainsi que l'analyse des supports ont été réalisés grâce à la collaboration du Laboratoire de Recherche des Monuments Historiques ${ }^{6}$. Ils nous ont éclairés sur les matériaux utilisés, confirmé les phases successives d'un même chantier à travers l'observation des couches picturales, séparées parfois par de fines traces de poussière ou dans le meilleur des cas par un fin badigeon.

\section{Données techniques : l'exemple du Sacrifice de Noé}

13 Les peintures recouvrant l'ensemble de la nef ont fait l'objet de compositions et de traitements très divers. Toutes ont cependant été réalisées selon le principe de la technique mixte et non pas à la fresque comme on l'a longuement soutenu.

Dans les trois premières travées, la couche picturale est posée directement sur un enduit appelé arricio ${ }^{7}$. Celui-ci n'est pas très bien taloché et laisse entrevoir les irrégularités du mur et parfois même les têtes des moellons. D'après le prélèvement effectué, il ne présente pas de trace d'un liant particulier : il est très chargé en sable et la chaux est le seul liant qu'on y trouve. L'épaisseur est très variable, pouvant aller de 1 
à $3 \mathrm{~cm}$. Cette épaisseur a une conséquence directe sur le temps de séchage du support : c'est la raison pour laquelle la première couche de peinture correspondant au dessin préparatoire est analysée comme ayant été posée a fresco. Toutes les autres couches picturales sont posées a secco. Le mur étant déjà sec, il n'y a plus de carbonatation possible.

La hauteur des registres sur lesquels se développent les scènes correspond à une pontata ${ }^{8}$, hauteur équivalente à celle d'un échafaudage qui a permis de mettre en place des personnages de dimensions supérieures à la taille humaine. Ce principe se rencontre également sur les peintures du porche et de la tribune, où les limites des pontate se placent exactement sous les pieds et sur les têtes des personnages, de manière à préserver les figures de toute coupure résultant d'une exécution en deux temps.

16 Ce n'est pourtant pas le cas des peintures de la nef réalisées au-delà du troisième doubleau. Dans ce cas précis, la hauteur correspond généralement à une pontata mais la pose des enduits se fait de façon plus grossière et les chevauchements des limites ne respectent pas les formes. Elles sont ondulantes et traversent parfois le torse ou les poitrines des personnages comme sur la scène des Tables de la loi ou de la Récompense de Joseph. Il est possible que cette pose peu soigneuse soit justifiée par la technique utilisée : une technique avec un badigeon intermédiaire qui permet de réunifier le support. L'ensemble de l'enduit est en effet recouvert par un badigeon bien taloché qui rend plus lisse la surface à peindre. C'est dans ce groupe de scènes que s'inscrit le Sacrifice de Noé.

17 La scène se développe sur une longueur d'environ 2,10 m, et se situe dans la logique du récit biblique : elle est précédée à gauche par la famille de Noé sortant de l'Arche et suivie à droite par Noé coupant sa vigne.

18 Lors d'une première observation, nous remarquons une composition simple: Noé est agenouillé sur une colline formée de pains de sucre et offre à Dieu deux colombes qu'il porte dans ses mains voilées. Un agneau se tient sur un autel accessible par une volée des petites marches. Dieu reçoit ces offrandes en faisant un signe bénédiction. Toute la partie inférieure de son corps ainsi qu'un bon pourcentage de la bande inférieure du registre à droite ont disparu et sont actuellement repris par des enduits modernes. Cette zone de la composition ne fournit donc aucune information.

19 La partie supérieure de la scène est également difficile à comprendre car nous avons plusieurs superpositions entre la bande faitière des XIVe et XVe siècles et la bande supérieure du registre réalisée au XIIe siècle. Celles-ci ont été remaniées avec des couleurs très proches, ce qui en complique l'analyse.

20 En dehors des cadres, l'état de conservation de la scène est suffisamment bon pour que l'étude de la technique et de la mise en couleur ait pu être réalisée. La scène est peinte avec une technique mixte sur badigeon'. Le support est constitué d'une couche de mortier d'environ $8 \mathrm{~mm}^{10}$, recouverte par un enduit à faible granulométrie ${ }^{11}$, sur lequel a été posé le badigeon de chaux destiné à recevoir la couche picturale ${ }^{12}$.

21 Les limites latérales sont marquées par des fils battus trempés dans du pigment rouge. Ces traces ont été reprises par des traits épais de couleur prune : à gauche, la trace du cordeau est perceptible sous le fond de la scène précédente. À droite, le tracé retenu se trouve à $8 \mathrm{~cm}$ au-delà de la limite initiale (sous le coude de Dieu). Les limites inférieure et supérieure ont été mises en couleur dès le début de la conception en même temps 
que le dessin préparatoire ${ }^{13}$. Elles sont en très mauvais état et se fondent actuellement dans les reprises postérieures du sol et de la bande faîtière. Grâce au relevé, nous avons pu observer qu'elles étaient composées de deux bandes horizontales jaune et rouge et d'une série de cercles blancs qui marquent la séparation entre les deux couleurs.

La scène présente une seule unité iconographique mais est divisée volontairement en trois zones verticales bien distinctes: blanche, verte et jaune. Elles se différencient ainsi des peintures des trois premières travées dans lesquelles les fonds également colorés $^{14}$ avaient pour fonction de marquer les limites iconographiques ou chronologiques des scènes. Ce qui est étonnant dans cette division est que le peintre n'a pas peint en vert l'ensemble du fond sur lequel se détache Noé afin de conserver une bipartition visuelle et d'encadrer chaque personnage avec une couleur. Il accentue la limite entre les fonds par un épais trait prune séparant ainsi visuellement Noé et Dieu qui font partie de la même scène.

La palette chromatique est ici très variée par rapport à celle utilisée dans les trois premières travées. Bien que les couleurs soient uniquement créées à partir de pigments naturels, les nuances sont plus recherchées : trois tons différents de rouge, dont un très foncé (que l'on appelle prune pour un souci de compréhension), un jaune clair et un jaune foncé, un mélange d'ocre rouge et jaune pour obtenir du rose et du saumon, un blanc, un vert et un gris.

Actuellement, il est impossible de savoir s'il existe dans les peintures de la nef de SaintSavin un autre liant organique que la chaux mélangée aux pigments ${ }^{15}$.

Les couleurs sont utilisées dans des pourcentages très différents et on remarque que le prune rehausse uniquement les ombres, que le blanc et le gris sont utilisés pour les lumières et que le jaune clair et le rose sont réduits aux aplats. Quant au vert, il a les deux fonctions : comme fond de l'image et pour les détails du cou de l'agneau. L'ordre d'application des couleurs observé par le chevauchement des pigments est: le jaune pâle, le rose, le rouge, le jaune foncé, le gris et enfin le prune et le blanc. On a donc appliqué une méthode qui rendait l'exécution plus rapide. Le travail a été achevé par l'application $\mathrm{du}$ fond vert.

Stratigraphie de l'élaboration (fig. $n^{\circ} 2$ ) 
Figure 2
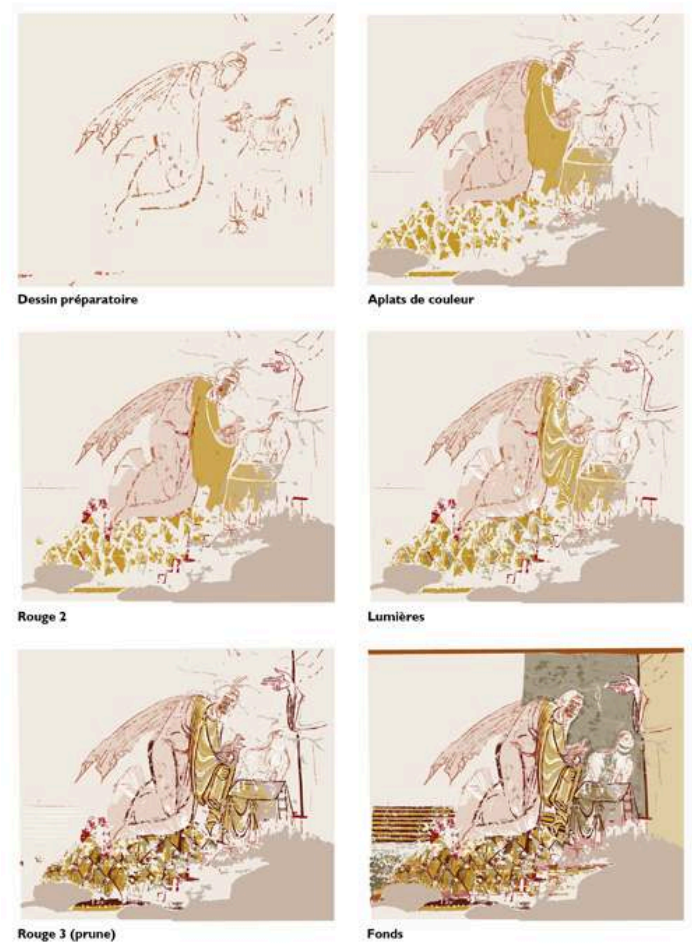

Saint-Savin-sur-Gartempe, peinture de la nef. Stratigraphie de la technique du Sacrifice de Noé. Relevé Sarrade, Carolina. (C) CESCM, décembre 2011.

1 - Badigeon blanc et rose ${ }^{16}$.

2 - Rouge clair, dessin préparatoire (Noé, agneau, partie inférieure de l'autel, nuée et main de Dieu).

3 - Aplats jaune et rose (vêtements, autel et montagne).

4 - Rouge foncé pour le dessin définitif (pieds, marches, quelques ombres, main de Dieu).

5 - Blanc et gris : lumières et rehauts (plis, motif à trois points sur la tunique de Noé), jaune foncé (rehauts de la montagne).

6 - Prune (ombres dans l'ensemble de la scène).

7 - Vert (fond).

8 - Bande de sol 2, inscription NOÉ et deuxième bande de registres.

On observe de multiples traces de dessin préparatoire pour la mise en place générale des peintures. Il est cependant impossible d'évaluer exactement leur étendue puisque les peintures ne sont pas usées de façon uniforme et leur transparence est variable. De plus, la plupart du temps les tracés préparatoires sont recouverts par les couches picturales postérieures ce qui rend leur lecture difficile. Le principe même de la technique en peinture veut que l'on recouvre l'esquisse avec le dessin final et qu'on l'atténue par l'application de couches successives. Nous avons donc identifié le dessin préparatoire sur la tête, la barbe, la cape et le bas de la tunique de Noé, sur les colombes, le dos de l'agneau, la partie inférieure de l'autel. Sur l'ensemble de la montagne en pains de sucre, nous n'avons pas réussi à trouver des traces du dessin 
préparatoire qui auraient pu éventuellement être recouvertes par les rehauts prune. Les aplats jaunes posés dans un deuxième temps se concentrent sur la tunique et la montagne. Ils sont mis en valeur par une série de rehauts blanc et gris qui créent le volume.

La tunique et la cape de Noé sont actuellement roses: elles n'ont pas une couleur spécifique car on leur a attribué la teinte de l'intonaco. Leur relief est uniquement créé par la juxtaposition de traits blancs, gris ${ }^{17}$ et rouges marquant les plis, comme pour Abel dans la scène du Sacrifice. Les plis rouge clair correspondent au dessin préparatoire visible par transparence. Ils ont été repris par le rouge foncé et le prune qui délimitent les bords de certains éléments comme les jambes et le ventre.

Sous l'ensemble de la surface, on trouve un léger badigeon rose dont la présence a été observée également par les restaurateurs sur d'autres scènes ${ }^{18}$. Il me semble qu'il ne s'agit pas d'une couche picturale mais plutôt d'un badigeon "rougi ou Sali » par les multiples usages des outils. Il occupe la même position stratigraphique que le fond blanc. Il est important de savoir qu'une couche picturale posée sur un badigeon de chaux blanchit en séchant sous l'effet de la carbonatation. Il est donc possible qu'en raison de la très légère différence de teinte du fond, le peintre ait compté sur le blanchissement de la paroi consécutive au séchage pour que cette erreur ne soit pas perceptible à 16 mètres de hauteur.

L'inscription " NOÉ »"19, apparaît actuellement en négatif. Les lettres, aujourd'hui roses, étaient à l'origine blanches sur fond vert. La couleur verte est tombée avec le temps et laisse transparaître actuellement la couleur du badigeon posé en sous-couche.

Le fond blanc du panneau, correspondant au badigeon du support, ne présente aucune trace de polychromie ni de lissage ${ }^{20}$. Le fond vert semble également homogène mais il borde certains éléments comme le dos et la tête de Noé, les colombes et l'agneau et recouvre parfois la ligne de séparation. Cette application montre que le fond vert a été posé en fin d'intervention selon un principe de nettoyage des fonds des scènes.

Le fond vert efface un peu la partie supérieure du manteau de Noé, en limitant et en bordant son visage. Il redessine les contours de l'agneau à une distance variable allant de quelques millimètres à deux centimètres. Le fond s'approche de la bande rouge verticale tout en laissant quelques vides blancs ou en la recouvrant. On peut donc en déduire que la pose $d u$ fond vert s'est faite d'une façon rapide et sans grande minutie, ce qui signifie que les peintres prenaient en compte la distance à laquelle allaient être observées les peintures.

41 Sous le fond vert de la scène se détachent néanmoins des traces rouges avec des formes variées. On y aperçoit, selon le même principe que dans Le Sacrifice de Caïn et Abel la main divine sortant d'une nuée. Cette observation a posé la principale problématique de cette scène. Ces traces correspondent-elles à un changement iconographique ? Quelle est la composition stratigraphique de la scène?

\section{Un changement iconographique volontaire ou une maladresse du peintre?}

42 À l'origine, la vendange de Noé aurait dû se situer à environ $60 \mathrm{~cm}$ vers la droite, sur l'ensemble du fond jaune où s'inscrit actuellement la figure de Dieu. Une série de branches de vigne perceptibles par transparence sous le fond jaune, la tête de Noééc, le 
dessin de la tunique de Noé sous celle de Dieu, la nuée et la main divine montrent qu'il $\mathrm{y}$ a eu un changement iconographique (fig. $\left.\mathbf{n}^{\circ} \mathbf{3}\right)$. Suite à un important repentir, le concepteur a en effet décidé de remplacer la main de Dieu sortant d'une nuée par la figure de Dieu debout, comme dans Le Sacrifice de Caïn et Abel. Il a donc été obligé de repousser la scène de la vendange vers la droite, autrement dit, vers l'extrémité orientale du registre.

Figure 3

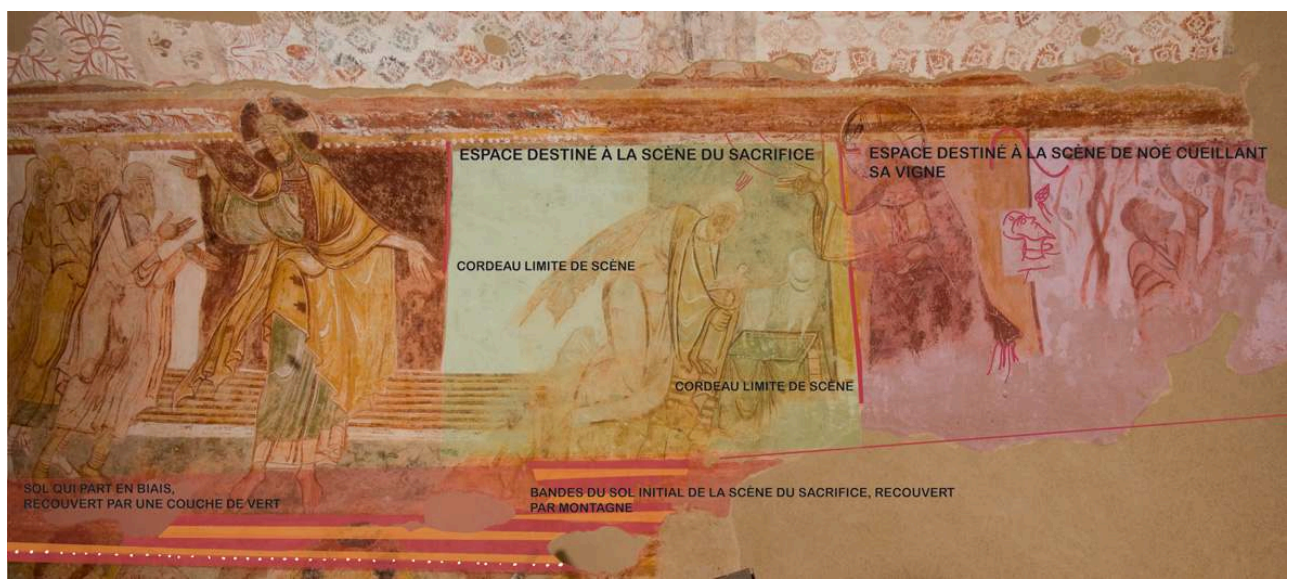

Saint-Savin-sur-Gartempe, peinture de la nef. Schéma des scènes reprises : Sortie de l'Arche, Sacrifice de Noé, Vendange de Noé.

Relevé Sarrade, Carolina. (C) CESCM.

Il semble toutefois qu'à l'inverse de la première version du Sacrifice de Caïn et Abel, la composition initiale du Sacrifice de Noé n'ait pas été mise en couleur. Si l'ensemble des éléments repris n'avait effectivement pas dépassé le stade du dessin préparatoire, on peut penser que le repentir serait intervenu très rapidement.

On rencontre également un changement de conception sur le tracé de la bande du sol initial et celle de registre inférieur : ces bandes semblent avoir été involontairement déviées de leur trajectoire horizontale. Elles partent en biais en s'élevant progressivement vers la droite et donc en rétrécissant de plus en plus l'espace destiné aux scènes qui devaient se dérouler vers l'est. Il a donc été nécessaire, comme l'ont suggéré les restaurateurs ${ }^{22}$, de retracer la trajectoire de cette horizontale avec une nouvelle bande inférieure et de recouvrir l'erreur initiale avec un nouveau sol. Il s'agit d'une importante modification effectuée avant la réalisation des scènes du registre inférieur ${ }^{23}$. La scène de Moïse recevant les Tables de la Loi a été exécutée une fois la scène supérieure modifiée puisqu'on remarque que les auréoles des anges couvrent la bande de registres tout en restant visibles par transparence.

Suite à la réalisation du relevé stratigraphique et suivant les traces de dessin préparatoire, nous avons observé que dans la première version du Sacrifice, le Mont Ararat n'était peut-être pas matérialisé car nous n'avons trouvé aucun vestige de dessin préparatoire. Noé aurait été simplement posé sur un sol bicolore composé d'horizontales jaunes et rouges, comme celui de l'Ivresse de Noé, et des marches qui mènent vers l'autel. Le sol posé sur la bande de registre initiale était déjà mis en couleur et suivait également la trajectoire diagonale de la bande inférieure. 

l'origine, destinées à représenter un sol végétal comme pour l'ensemble des autres scènes situées sur le registre supérieur de la voûte à l'est du faux-doubleau ${ }^{24}$. L'emploi de cette couleur foncée sur le sol est-il justifié par l'erreur d'emplacement de la première bande de registre? A-t-on cherché à cacher cette maladresse ? Cette question se pose avec acuité car, pour le reste des scènes représentées à l'extérieur, le sol végétal sur lequel sont placés les personnages est représenté non pas par la couleur verte mais par des ondulations fleuries.

47 Le sol vert et les montagnes viennent cacher la première bande de séparation de registre initialement plus haute et la deuxième bande de registre est peinte sous la montagne.

Les traces du dessin préparatoire posées sur le badigeon en première strate sont visibles sous les figures de Noé, des colombes, de l'agneau et sous l'autel, suggérant ainsi qu'ils étaient présents dès le début de la conception, et qu'il n'y a pas de changements correspondant à ces éléments.

49 Le manteau soufflé par le vent pose en revanche quelques problèmes d'analyse. Il est rendu uniquement par le dessin préparatoire, sans la moindre couche picturale. Il est possible que le traitement stylistique du manteau par transparence ait été identique à celui du voile sur lequel Abel porte l'agneau dans la scène du sacrifice ${ }^{25}$. L'ensemble du manteau semble avoir été gratté, des petites traces en relief d'une couche gris blanc et rose épaisse recouvrant parfois sa surface. Cette couche se trouve sur la strate correspondant à la tunique rose de Noé mais elle ne présente pas une harmonie stratigraphique très claire et pose quelques problèmes d'interprétation. A-t-elle été dégagée? À quel moment? L'effacement correspond-il à une usure de la couche picturale blanche?

On pourrait être tenté de croire qu'il s'agit d'un dégagement puisque le type d'usure est peu uniforme et semble partiellement réalisé. Il me semble que cette cape fait partie de la conception initiale de l'œuvre. Ses traits appartiennent au dessin préparatoire a fresco que l'on aurait voulu effacer avec une couche de badigeon sur un enduit déjà plus sec. Ainsi la cape serait-elle restée toujours un peu perceptible par transparence ${ }^{26}$. Cette hypothèse est confirmée par le fond vert qui recouvre le pourtour du dos de Noé et efface le manteau sur la partie supérieure. La partie inférieure du dos est redélimitée par un large tracé prune recouvrant la cape vers le haut selon le même principe (fig. $\left.\mathbf{n}^{\circ} \mathbf{4}\right)$. 
Figure 4

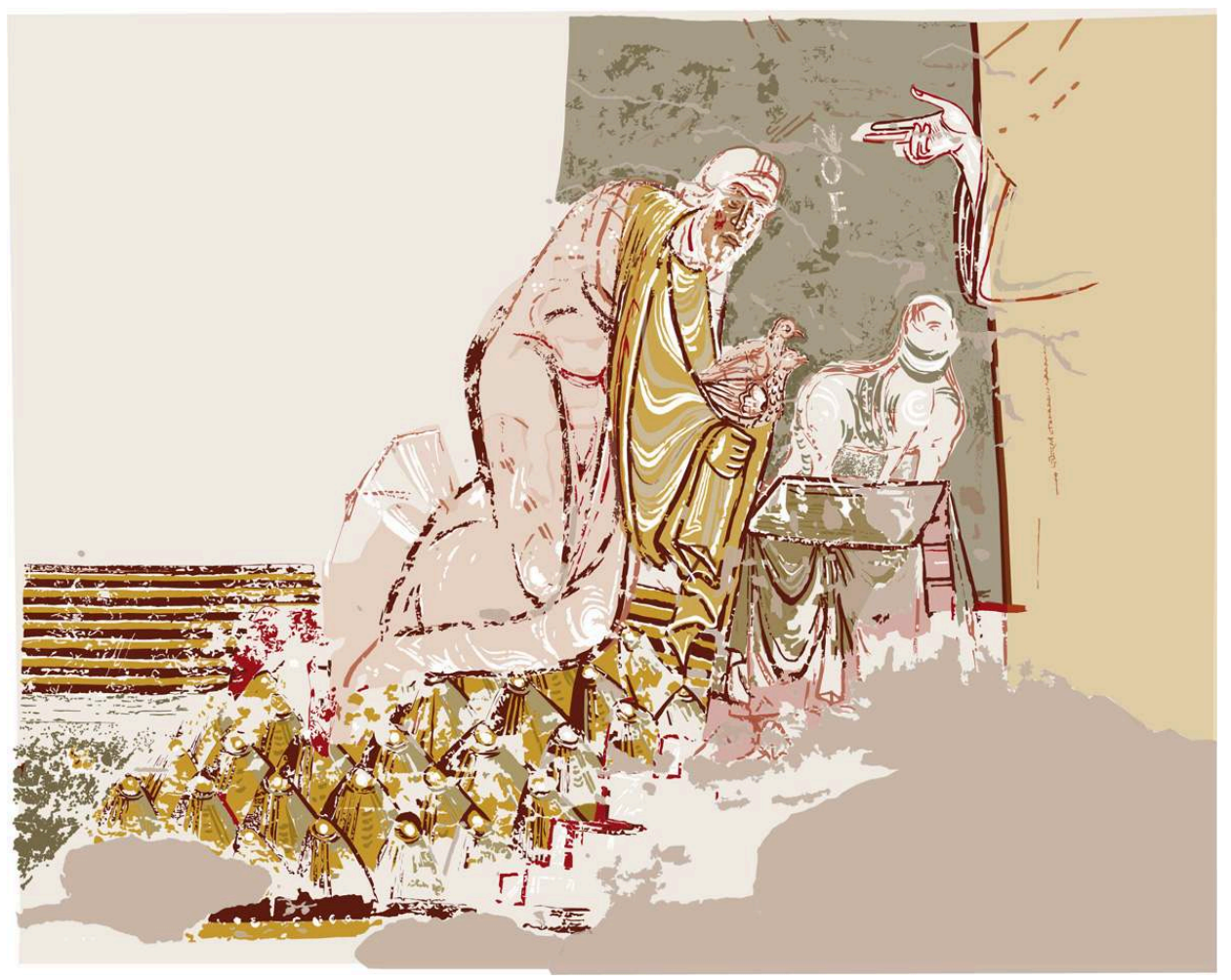

Saint-Savin-sur-Gartempe, peinture de la nef. Essai de restitution, Noé sans cape.

Phot. Sarrade, Carolina. (C) CESCM décembre 2011.

51 Ce repentir soulève la question de la volonté de faire disparaitre le premier manteau soufflé par le vent et de garder celui que Noé enroule autour de son bras. Il est possible que le concepteur ait voulu voiler les mains de Noé de la même manière que dans Le Sacrifice de Caïn et Abel. Il aurait alors fait disparaitre le premier manteau pour éviter le doublement de ce type de vêtement.

Le manteau soufflé de Noé ne présente aucune trace de polychromie et semble homogène. Il faudra donc s'interroger sur les motivations d'un tel choix, d'autant que ce vêtement a peut-être été effacé dans un second temps. Dans l'affirmative, le panneau blanc serait resté entièrement vide. La bande faitière a fait l'objet d'interventions à la fin du Moyen Âge. Nous connaissons d'autres exemples de reprises effectuées sur des éléments qui lui sont proches (visages, auréoles). La cape aurait donc pu être dégagée lors d'une de ces interventions.

\section{Conclusion}

Une première approche de la scène du Sacrifice de Noé révèle la présence de la main de Dieu simplement esquissée ainsi que le chevauchement de couches picturales dans la représentation du sol. L'analyse stratigraphique nous a permis de décomposer ces strates et de mieux comprendre les deux étapes de la composition.

Dans un premier temps, on a tracé l'esquisse d'une composition simple dans laquelle le sol était constitué de traits obliques sur lesquels venait s'agenouiller Noé offrant à la main divine deux colombes enveloppées dans un pan de son manteau, devant un autel 
desservi par des petites marches et sur lequel se dressait un agneau. Une première modification de la scène a été motivée par une mauvaise orientation du sol initial qui s'élevait progressivement en s'approchant de l'extrémité orientale de la nef, rétrécissant de près d'un mètre la hauteur de la Vendange de Noé.

Pour rattraper cette maladresse on a recouvert le sol initial d'un nouveau sol vert foncé orné de fleurs blanches et une montagne en forme de pains de sucre sur laquelle est venu se poser Noé. Et, sans doute dans le même temps, on a remplacé la main de Dieu par la figure entière comme on l'a fait pour Le Sacrifice de Caïn et Abel.

Le Sacrifice de Noé est une scène très riche au niveau iconographique, mais aussi au niveau technique, son analyse nous a permis de décomposer parfaitement les strates qui composent une peinture romane, sa technique est comparable par sa simplicité aux scènes des trois premières travées, même si son style reste différent et serait plutôt à rapprocher de l'ensemble du Cycle de Noé.

\section{NOTES}

1. - Édifices et peintures aux IV $V^{e}-X I^{e}$ siècles. Actes du 2e colloque du CNRS Archéologie et enduits peints, 7-8 novembre 1992, Auxerre, Abbaye Saint-Germain. Auxerre: Musée d'Auxerre, 1994. ROLLIER-HANSELMANN, Juliette. «D'Auxerre à Cluny : techniques de la peinture murale entre le $\mathrm{VIII}^{e}$ et le XII ${ }^{e}$ siècle en Bourgogne ». Cahiers de Civilisation médiévale, 1997, t. 40, p. 57-90. SAPIN, Christian (dir.). Peindre à Auxerre au Moyen Âge, IV -XIVe siècle. Dix ans de recherches à l'abbaye SaintGermain et à la cathédrale Saint-Étienne d'Auxerre. Paris : Éditions du CTHS, 1999.

2. - BESSAC, Jean-Claude, BURNOUF, Joëlle, JOURNOT, Florence, PRIGENT, Daniel, SAPIN, Christian, SEIGNE, Jacques. La construction, la pierre. Paris: Collection Archéologiques, 1999. PARRON-KONTIS, Isabelle, REVEYRON, Nicolas (dir.). Archéologie du bâti. Pour une harmonisation des méthodes. Actes de la table ronde des 9-10 novembre 2001. Paris : Musée archéologique de SaintRomain-en-Gal, 2005. COUTELAS, Arnaud (dir.). Le Mortier de chaux. Paris: Collection Archéologiques, 2009.

3. - Cette méthode de fixation ne peut s'appliquer que sur des peintures déjà consolidées. Elle pose d'ailleurs le problème de l'impossibilité d'intervenir sur ces structures particulièrement fragiles. Dans la crypte de Saint-Nicolas de Tavant (Indre-et-Loire), la localisation des peintures sur les voûtes a contraint à utiliser une structure en bois soutenant le calque. À Saint-Savin, ce dispositif n'était pas envisageable car il aurait provoqué un encombrement excessif du chantier. Aussi a-t-on opté pour la solution des calques fixés sur les enduits modernes, déjà prônée à SaintGermain ou à la cathédrale d'Auxerre ainsi qu'à l'église de Branches (Yonne).

4. - FAVREAU, Robert. Saint-Savin, l'abbaye et ses peintures murales. Poitiers: Connaissance et promotion du patrimoine de Poitou-Charentes, 1999.

5. - La technique utilisée par le LRMH - la spectrométrie Raman faite sous microscope - présente le grand avantage de permettre l'étude des fragments de dimensions microscopiques pouvant être apportés au Laboratoire. En raison de leur infime dimension, l'œuvre reste ainsi quasiment inaltérée. Pour la nef de Saint-Savin, les analyses ont été faites exclusivement sur de nouveaux prélèvements, bien localisés, qui sont venus compléter les échantillons déjà effectués lors de la restauration précédente (1969) et ont permis une étude comparative des résultats. Le choix des 
emplacements des micro-prélèvements lors de cette nouvelle intervention a suivi les indications des restaurateurs et des archéographes afin de sélectionner les décors susceptibles de répondre aux problématiques soulevées. Le choix est toujours contraint par le risque de mettre en péril un décor : il n'y a donc eu aucun prélèvement sur les visages et les mains des personnages.

6. - Ils ont été réalisés par Paulette Hugon et Dominique Martos, chargées de l'étude (équipe de peintures murales du LRMH, Champs-sur-Marne) que je tiens à remercier ici pour leur collaboration et leur enseignement. Plusieurs échantillons de chaque couleur ont été prélevés dans l'ensemble des scènes et ont été analysés au microscope électronique à balayage qui, en complément de l'étude au microscope optique, s'est avéré indispensable pour déterminer la nature chimique des composants inorganiques constituant les pigments et établir avec certitude la superposition des différentes couches picturales.

7. - Mortier préparé à base de chaux et de sable que le peintre étalait sur le mur tout en le laissant rugueux. La chaux était utilisée comme liant en raison de ses capacités de conservation des pigments. Les proportions de sable et de chaux étaient variables suivant la finesse voulue pour le support, néanmoins la dernière couche qui reçoit la peinture est la plus lisse.

8. - Plans souvent rectangulaires que l'on détermine par rapport à la surface de mur où les peintres travaillent sur un échafaudage. Dans la peinture romane, on constate que les artistes avaient pour tradition de travailler selon un système à pontata, la surface à peindre étant divisée en larges zones correspondant à la hauteur de l'échafaudage (ponteggio en italien). Les limites des joints entre ces pontate se trouvent généralement en bordure des scènes, mais souvent aussi au niveau des genoux des personnages. Elles peuvent être décelées aisément en lumière rasante. Le travail en pontate devait être relativement rapide, puisque la surface à enduire correspondait à toute une scène.

9. - Confirmée par les strates des prélèvements analysés par le LRMH et par les observations des restaurateurs.

10. - Épaisseur observée lors de la consolidation de la bande faîtière qui surmonte la scène.

11. - Les principales caractéristiques que l'on peut observer sur un enduit sont la couleur de la pâte, la texture, la porosité, la teneur, les vides et la granulométrie. Cette analyse nous informe également sur la mise en œuvre de l'enduit et la préparation de la surface qui reçoit le badigeon ou les pigments.

12. - Appelée plus précisément intonaco (nom italien) qui recouvre l'arricio et qui est celui qui reçoit la couche picturale.

13. - Rouge et jaune, sans rehaut blanc final.

14. - Les fonds étaient posés en fin d'intervention, donc sur un enduit presque sec. Ils étaient alors bien plus fragiles et ont complètement disparu. Lors de la dernière restauration, l'observation minutieuse des restaurateurs a motivé des prélèvements qui confirment la coloration initiale des fonds.

15. - Les anciennes restaurations ayant été traitées avec une surabondance de produits de fixation empêchent actuellement des résultats fiables et il est impossible d'identifier les liants organiques. Néanmoins, en 2008, lors de la dernière restauration, Claire Dandrel a pu identifier des restes de petites figures originales cachées sous l'enduit XIX ${ }^{\mathrm{e}}$ sur l'intrados d'un arc. Malheureusement, sa requête d'identification de liant n'a pas pu être réalisée mais il devrait être possible de répondre à cette question dans un avenir proche.

16. - Le ton rose occupe le même niveau stratigraphique que le badigeon blanc. Il me semble qu'il pourrait s'agir d'un mélange de pigments qui se serait produit sur un pinceau lors de la pose.

17. - Le gris est une couleur qui est utilisée en tant que telle, parfois même ravivée par l'ajout de tons rouges.

18. - Scènes allant au-delà du faux doubleau vers l'est. 
19. - DEBIAIS, Vincent. Étude des inscriptions de la nef de Saint-Savin-sur-Gartempe. Rapport d'intervention, juin 2008. Poitiers : CESCM-CNRS UMR 7302, 2011, p. 14.

20. - STEFANNAGGI, Marcel. Les techniques de la peinture murale. Laboratoire de recherche des Monuments historiques. Champs-sur-Marne: LRMH, 1997. Remarque dans la technique mixte une disparition progressive du lissage de surface.

21. - Dégagée par Marie-France de Christen dans les années 1970.

22. - Les restaurateurs ont observé une couche de "masquage » sur la scène précédente. Brice Moulinier attribue ce changement à la modification totale d'une première bande de registres déjà mise en place et qui aurait été tracée en biais, conditionnant ainsi la place attribuée au Sacrifice de Noé. Il y aurait donc eu effacement d'une première bande de registres et mise en place d'une deuxième limite inférieure pour la scène. La couche de "masquage » est donc associée à la modification des scènes, suite à la correction de la bande de registre.

23. - Scènes 54 et 55 où l'on aperçoit le chevauchement des deux étapes d'intervention sur les auréoles des anges.

24. - Caïn tuant Abel, Dieu maudissant Caïn, Enlèvement d'Enoch, Sortie de l'Arche et Sacrifice de Noé.

25. - SARRADE, Carolina. Étude archéographique des peintures de la nef de Saint-Savin-sur-Gartempe. Rapport Décembre 2011. Poitiers : CESCM-CNRS UMR 7302, p. 19.

26. - Ce type d'intervention pourrait être comparable à l'emploi d'une couche de nettoyage finale, technique connue dans les peintures romanes, comme par exemple dans la crypte de l'église de Saint-Nicolas de Tavant. CHRISTEN, Marie-France (de). « La technique des peintures de la tour-porche et de la nef : couleurs, traces, repentirs et restaurations ». Dans FAVREAU, Robert. Saint-Savin, l'abbaye et ses peintures murales. Poitiers : Connaissance et promotion du patrimoine de Poitou-Charentes, 1999, p. 150.

\section{RÉSUMÉS}

Le relevé stratigraphique met à la disposition du chercheur une nouvelle méthode d'analyse des peintures murales. Le travail consiste à étudier la technique, à proposer un résumé graphique des différents stades d'élaboration et à déterminer une chronologie relative des décors dans l'ensemble du programme pictural. Le décalque extrêmement fidèle donne naissance à une reproduction entière de l'œuvre à taille réelle sur laquelle il est possible de restituer l'état originel et de combler certaines lacunes iconographiques sans intervenir directement sur le mur.

The stratigraphic drawing provides to the researcher a new analysis method for murals paintings. This consists on a study of the technic, a proposal of a graphical summary of the various developments stages and a determination of a relative chronology of the decoration in the whole pictorial program. The extremely faithful tracing gives rise to an entire reproduction of the work in actual size in which it is possible to restore the original state and fill some iconographic gaps without intervening directly on the wall. 
INDEX

Mots-clés : peinture murale, relevé archéographique, décomposition stratigraphique, technique picturale

\section{AUTEUR}

\section{CAROLINA SARRADE}

Ingénieur d'études - CNRS Centre d'Études Supérieures de Civilisation Médiévale (CESCM) - UMR 7302 Université de Poitiers carolina.sarrade@univ-poitiers.fr 